\title{
Studies of the ultra-high energy cosmic ray composition at the Pierre Auger Observatory
}

\author{
Michael Prouza* for the Pierre Auger Collaboration ${ }^{\dagger}$ \\ Institute of Physics of the Academy of Sciences of the Czech Republic, Praha, Czech Republic \\ E-mail: prouza@fzu.cz
}

The hybrid Pierre Auger Observatory, consisting of fluorescence detector with 24 telescopes and of surface detector with more than 1600 water Cherenkov stations, has been collecting high quality data since January 2004. In this contribution we present namely current results of studies of ultra-high energy cosmic ray composition and related results concerning the energy spectrum of these particles. The depth of maximum of air showers determined using fluorescence telescopes and the magnitude of the fluctuations of this maximum depth are reported as functions of energy. The results are compared with predictions of several hadronic interaction models. Then the other mass sensitive parameters from the measurements of the surface detector are briefly discussed. Finally, we also mention the new limits on the flux of ultra-high energy photons and of diffuse ultra-high energy neutrinos, and we investigate the impact of these limits on top-down models of cosmic ray origin.

The 2009 Europhysics Conference on High Energy Physics,

July 16 - 222009

Krakow, Poland

\footnotetext{
* Speaker.

${ }^{\dagger}$ The author list of the Collaboration is available online: http://www.auger.org/admin/Collaborators/author_list_alphabetical.html.
} 


\section{Introduction}

The study of cosmic rays is one of the oldest branches of particle physics. First observations of cosmic rays were carried out at the very beginning of the twentieth century, and the extraterrestrial nature of these extremely energetic particles is known already for almost one hundred years. However, the most fundamental questions still remain unanswered, especially for the region of the most energetic particles known in the universe - for the ultra-high energy cosmic rays ${ }^{1}$. We still do not know what is the character and the composition of these enigmatic particles, and from which type (or types) of sources they come from.

The Pierre Auger Observatory, which significantly surpasses all previous ultra-high cosmic ray detectors in many aspects, most notably in the area covered and in the integrated exposure, finally starts to provide first answers to a century-old fundamental questions in particle physics.

\section{Pierre Auger Observatory}

The Pierre Auger Observatory is currently the largest and the most complex detector of the ultra-high energy cosmic rays. It is also the first detector using the concept of so-called hybrid detection. As an hybrid detector, the Pierre Auger Observatory combines two independent detection subsystems, an array of surface detectors and a set of fluorescence telescopes. The use of two independent detection techniques then significantly improves the quality and precision of the data analysis. Hybrid detection enables a combination of advantages of both techniques and an omission of their drawbacks.

More specifically, the measurement using fluorescence radiation of the cosmic ray showers is almost calorimetric and it also allows the observation of the longitudinal development of the showers. On the other hand, the surface detector has very well determined aperture. Using the data obtained by fluorescence telescopes, well chosen events serve for calibration of the observables of the surface detector. This calibration is then used for determination of the energy of all surface detector events, and thus to construct the precise energy spectrum.

The Pierre Auger Observatory was conceived as the full-sky detector, consisting of two sites, one on each hemisphere. The southern site is located close to the town Malarguie in Mendoza province in Argentina $\left(69^{\circ} \mathrm{W}, 35^{\circ} \mathrm{S}\right.$, altitude $\sim 1400 \mathrm{~m}$ ), and its construction began in 2003 and was completed in 2008. The northern site is still in its R\&D phase and should be located close to the town Lamar in Colorado, USA.

The area of the southern site is defined by the roughly rectangular array of water Cherenkov surface stations, which covers $\sim 3000 \mathrm{~km}^{2}$. It consists of more than 1600 individual stations that are distributed in a regular hexagonal grid with $1.5 \mathrm{~km}$ separation between neighboring stations. This large array is overlooked by four fluorescence detector stations, each of which is situated at the array border and each is composed of six individual fluorescence telescopes.

Each surface detector tank consists of a large plastic vessel that contains 12000 litres of pure water. Secondary particles of cosmic ray showers pass through the water and produce Cherenkov light that is detected by three large photomultipliers.

\footnotetext{
${ }^{1}$ For the purposes of this article we define ultra-high energy cosmic rays as particles with energy above $10^{18} \mathrm{eV}$.
} 
Each fluorescence telescope is based on the design of the Schmidt camera, and has field of view of $30^{\circ} \times 30^{\circ}$. It is composed of a very large segmented mirror of total area of $11 \mathrm{~m}^{2}$, and a camera of 440 photomultipliers.

The detailed description of the fluorescence detector is available in [1] and of the surface detector in [2].

\section{Energy spectrum}

Naturally, the first result of the Pierre Auger Observatory that we have to mention is the energy spectrum [3]. In general, the spectrum of the cosmic rays is steeply falling, and according to theoretical expectations of the Greisen-Zatsepin-Kuzmin cutoff [4], it should be strongly suppressed in the region above $10^{19.5} \mathrm{eV}$. For several years during the last decade this was a source of the controversy of the most important projects in this field - between Japan experiment AGASA [5] (which did not see the cutoff) and of U.S. experiment HiRes [6] (which did see the cutoff).

Now, with the largest statistics ever obtained, the measurement at the Pierre Auger Observatory strongly confirms the existence of the suppression in the spectrum (see also Fig. 1). The spectrum can be measured independently using surface detector events, hybrid events (measured simultaneously by surface and fluorescence detectors), or using very inclined showers (zenith angle $>60^{\circ}$ ) only. All these different types of spectra are in good agreement [7].
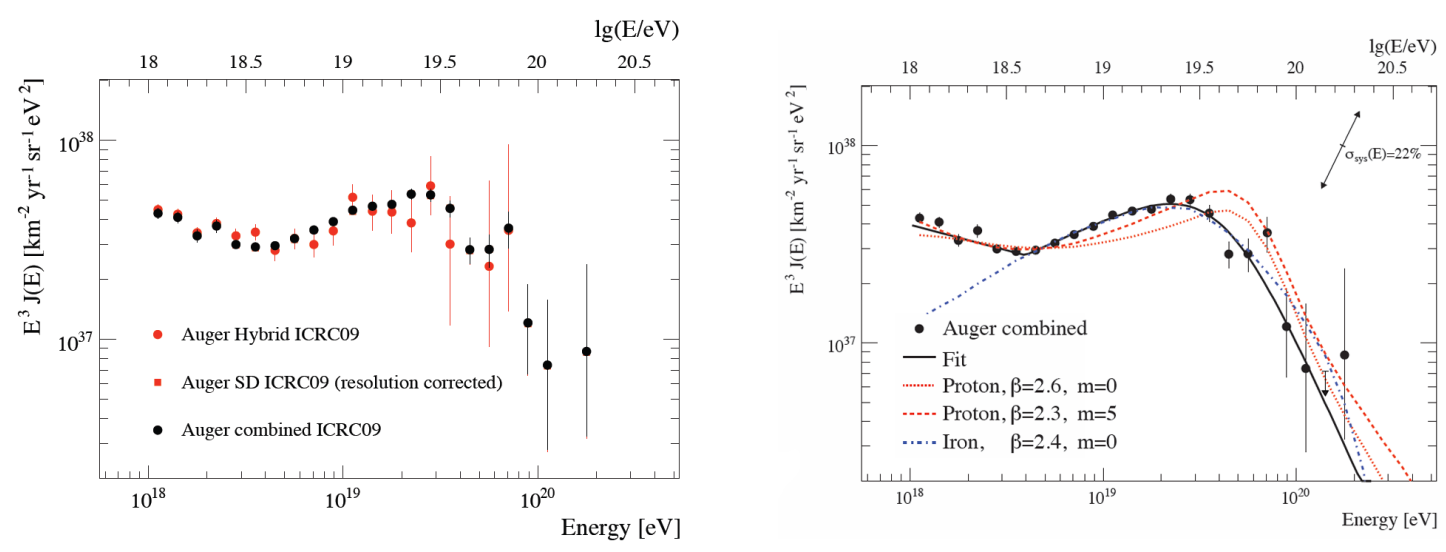

Figure 1: The energy spectrum of the Pierre Auger Observatory. The left panel shows the spectra based on hybrid events, on surface events, and on their likelihood combination. The right panel compares the combined spectrum with several source models, which follow the cosmological evolution of the source luminosity of $(z+1)^{m}$. From [7].

\section{Composition}

Another important result of the Pierre Auger Observatory, which was significantly updated only very recently [8], is the measurement of the cosmic ray composition. As no photons or no neutrinos were detected yet (see Sec. 5), we can expect the hadronic nature of the incoming particles. Furthermore, although according to some authors [9] neutrons can be accelerated to ultra-high 
energies, they are unstable and will decay (neutron with energy $10^{20} \mathrm{eV}$ will decay after $0.9 \mathrm{Mpc}$ ) [10], and thus they can not travel across the extragalactic distances. Therefore we usually assume that the ultra-high energy particles are charged and may be protons or any other heavier nuclei up to the mass of iron.

The composition of the ultra-high energy cosmic rays may be studied using longitudinal shower profiles that are available from the observations of the fluorescence detector. The position of the average shower maximum $\left(\left\langle X_{\max }\right\rangle\right)$ and also the shower-to-shower fluctuations of the shower maximum $\left(\operatorname{RMS}\left(X_{\max }\right)\right)$ are sensitive parameters that indicate the average mass of cosmic rays. The basic idea behind both methods of mass determination is simple. Because of the larger cross-section, any heavier nucleus interacts on average earlier, ie. higher in the atmosphere. And due to the "averaging" over many nucleons in a heavy nuclei, the fluctuations are also smaller for the heavy nuclei. More details are available in [8].

The results from the surface detector may be also used for the composition analysis. Here, we do not have available the longitudinal shower profile, and thus we use other mass sensitive parameters such as e.g. risetime ${ }^{2}$. The time profile of particles reaching the ground is sensitive to cascade development — the higher the production height, the narrower is the time pulse [11]. The first portion of the signal is dominated by the muon component, which arrives earlier and over a shorter period of time than electromagnetic particles. Since a number of muons with respect to electromagnetic component is sensitive to primary particle type and distance from shower maximum, parameters corelating with $X_{\max }$ can be constructed using measured risetimes in individual stations of the surface. It was proven in [11] that two parameters correlate particularly well with the position of the shower maximum - the deviation to average risetime and also the risetime asymmetry. More details are again available in [11].
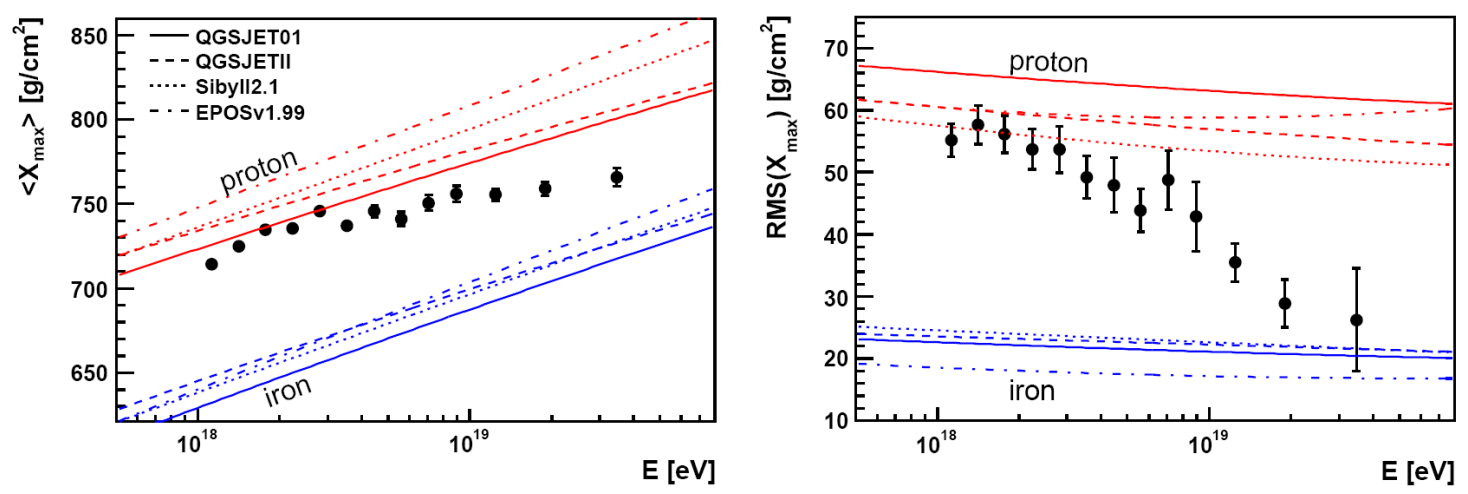

Figure 2: The left panel shows the dependence of the position of the average shower maximum $\left\langle X_{\max }\right\rangle$ on the energy in comparison with predictions of several models of hadronic interactions. The right panel shows the shower-to-shower fluctuations of the shower maximum ( $\operatorname{RMS}\left(X_{\max }\right)$ ), again in comparison with models of hadronic interactions.

Currently, the results of the Pierre Auger Observatory indicate that the composition of the

\footnotetext{
${ }^{2}$ Risetime is the length of the time window, which starts when $10 \%$ of integrated signal is measured by a given station, and which ends when $50 \%$ of the integrated signal is detected.
} 
ultra-high energy cosmic rays evolves with increasing energy from the light nuclei to heavy nuclei (see Fig. 2). This is a quite surprising result, because almost all currently discussed models expected protons at the highest energies. Moreover, trajectories of heavy nuclei will be much more significantly curved, and therefore not pointing towards theirs sources. However, it must be stressed that such interpretation of data relies on the prediction of hadronic interaction models describing interactions at energies far from those currently available at accelerators and the correct understanding of mass composition will be likely delayed until the LHC data will cross-check the validity of these models.

\section{Other results}

The Pierre Auger Observatory already achieved a number of other very interesting results. Here we want only to mention the most stringent limit on the photon fraction in the ultra high energy cosmic rays (see Fig. 3 and [12]) and a very interesting limit on the flux of ultra-high energy neutrinos (Fig. 3 and [13]). Last, but not least observation fact that we can mention here is the discovered anisotropy of the particles with the highest energies ( $\gtrsim 60 \mathrm{EeV})$. The correlation with the cosmologically nearby active galatic nuclei (redshift $z<0.018$ ) reported in [14] in 2007 is weaker with the new data added. However, the isotropic distribution of the cosmic ray arrival directions is still excluded on a 99\% confidence level, and new interesting regions (most importantly Centaurus A) with interesting overdensities start to emerge.

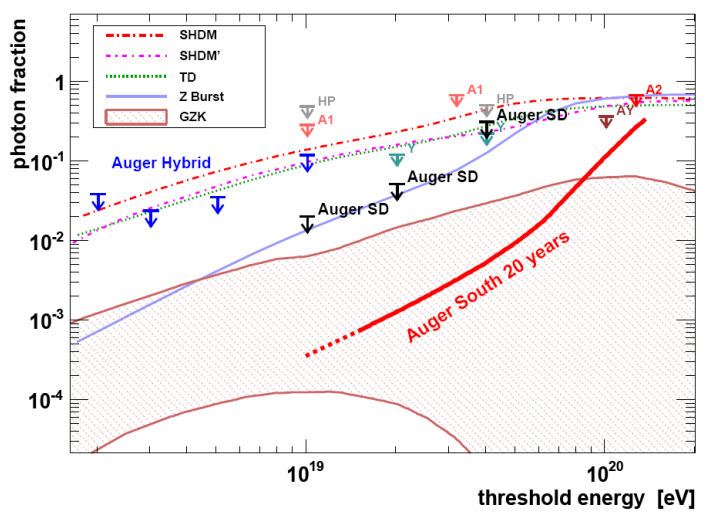

Single flavour neutrino limits $(90 \% \mathrm{CL})$

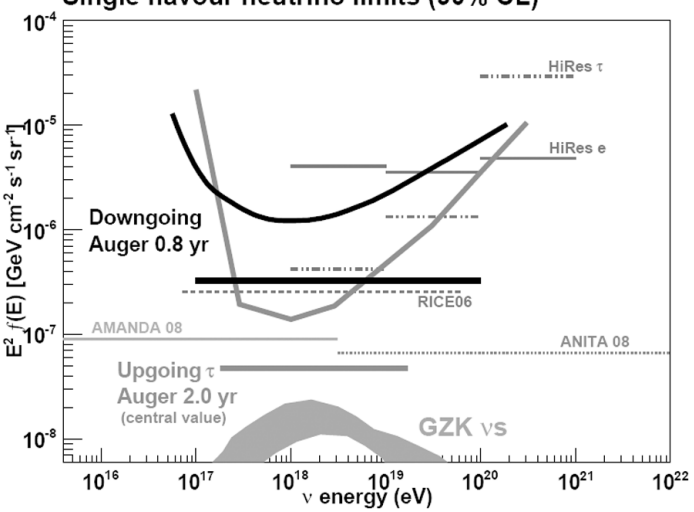

Figure 3: The left panel shows the upper limit on the photon fraction in the integral cosmic ray flux from various cosmic ray experiments (A denotes AGASA, Y denotes Yakutsk experiment and HP is for Haverah Park experiment). Some limits from the 'top-down' models of the ultra-high energy cosmic rays origin are also shown. Finally, the flux of cosmogenic photons, that are created via the GZK mechanism, is displayed too. From [12]. The right panel shows differential and integrated upper limits (90\% C.L.) from the Pierre Auger Observatory for a diffuse flux of down-going $v \mathrm{~s}$ in the period 1 Nov $07-28 \mathrm{Feb}$ 09, and of upgoing $v_{\tau}$ (1 Jan $04-28$ Feb 09). Limits from other experiments are also plotted, as well as a theoretical flux for GZK neutrinos. 


\section{References}

[1] J. Abraham et al. [Pierre Auger Collaboration] (2009), submitted to Nucl. Inst. Meth. A, also e-print: arXiv:0907.4282.

[2] I. Allekotte et al. [Pierre Auger Collaboration] (2008), Nucl. Inst. Meth. A. Nucl. Instrum. Meth. A586, 409.

[3] J. Abraham et al. [Pierre Auger Collaboration] (2008), Phys. Rev. Lett., 101:061101.

[4] K. Greisen (1966), Phys. Rev. Lett. 16, 748.

G. T. Zatsepin \& V. A. Kuzmin (1966), Zh. Eksp. Theor. Fiz. (Pisma Red.) 4, 114.

[5] Takeda M. et al. (2003), Astropart.Phys. 19, 447, also e-print arXiv:astro-ph/0209422.

[6] R. U. Abbasi et al. [The HiRes Collaboration] (2005), Phys.Lett. B619, 271, also e-print arXiv:astro-ph/050131.

[7] F. Schüssler [Pierre Auger Collaboration] (2009), Proc. 31th Int. Cosmic Ray Conf. (Lodz, Poland), also e-print arXiv:0906.2189.

[8] J. Bellido [Pierre Auger Collaboration] (2009), Proc. 31th Int. Cosmic Ray Conf. (Lodz, Poland), also e-print arXiv:0906.2319.

[9] C. Dermer (2002), Astrophys. J. 574, also e-print arXiv:astro-ph/0005440.

[10] P. Bhattacharjee, G. Sigl (2000), Phys. Rept. 327, 109, also e-print arXiv:astro-ph/9811011.

[11] H. Wahlberg [Pierre Auger Collaboration] (2009), Proc. 31th Int. Cosmic Ray Conf. (Lodz, Poland), also e-print arXiv:0906.2319.

[12] P. Homola [Pierre Auger Collaboration] (2009), Proc. 31th Int. Cosmic Ray Conf. (Lodz, Poland), also e-print arXiv:0906.2347.

[13] J. Tiffenberg [Pierre Auger Collaboration] (2009), Proc. 31th Int. Cosmic Ray Conf. (Lodz, Poland), also e-print arXiv:0906.2347.

[14] J. Abraham et al. [Pierre Auger Collaboration] (2007), Science, 318, 938.

[15] J. D. Hague [Pierre Auger Collaboration] (2009), Proc. 31th Int. Cosmic Ray Conf. (Lodz, Poland), also e-print arXiv:0906.2347. 